\title{
Interface Device
}

National Cancer Institute

\section{Source}

National Cancer Institute. Interface Device. NCI Thesaurus. Code C50008.

Either a program that controls a display for the user and that allows the user to interact with the system or a computer circuit consisting of the hardware and associated circuitry that links one device with another. 